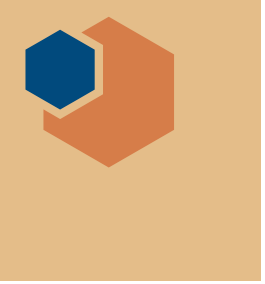

\section{US Department of Defense funds high-risk innovative materials research}

\author{
www.grants.gov
}

$\mathrm{N}$ ext to celebrities and superstar athletes, a sure-fire way to draw a crowd on Capitol Hill in Washington, DC, lately is to present some amazing scienceincluding materials research. That was certainly the case on January 30 at a luncheon briefing for members of Congress and their staff, put on by the Coalition of National Security Research (CNSR), an advocacy group for sustained support for the US Department of Defense (DoD) science and technology (S\&T) research programs administered by the Pentagon's Basic Research Office (BRO).

Along with a poster session - and a great boxed lunch - a panel of researchers talked about their work in new or emerging areas of science that has been funded by the Pentagon because of its potential applications and benefits for the military. The speakers and other presenters were representatives from CNSR's broad alliance of research universities and institutes, scientific and professional organizations, and other nonprofits that advocate for a strong defense S\&T enterprise.
The briefing's wow factor began with Neil Gershenfeld, director of Massachusetts Institute of Technology's Center for Bits and Atoms - a laboratory dedicated to breaking down the barriers between the digital and physical worlds. He spoke of the Pentagon's quest to reduce its supply chain to just 20 parts and how his concept of "digitizing fabrication" might help.

Gershenfeld said he began with the concept of amino acids being the basic 20 parts of human beings.

"We are trying to do the same things with nonbiological materials," Gershenfeld said. With a discreet set of parts that can be reversibly joined, "we could merge computation, communications, and fabrication." And rather than three-dimensional printers, the system he envisions would utilize self-reproducing assemblers, which could ramp up production of desired objects exponentially.

Gershenfeld has labeled his concept in situ resource utilization. The practicality of being able to build materials on the battlefield with a discreet set of building

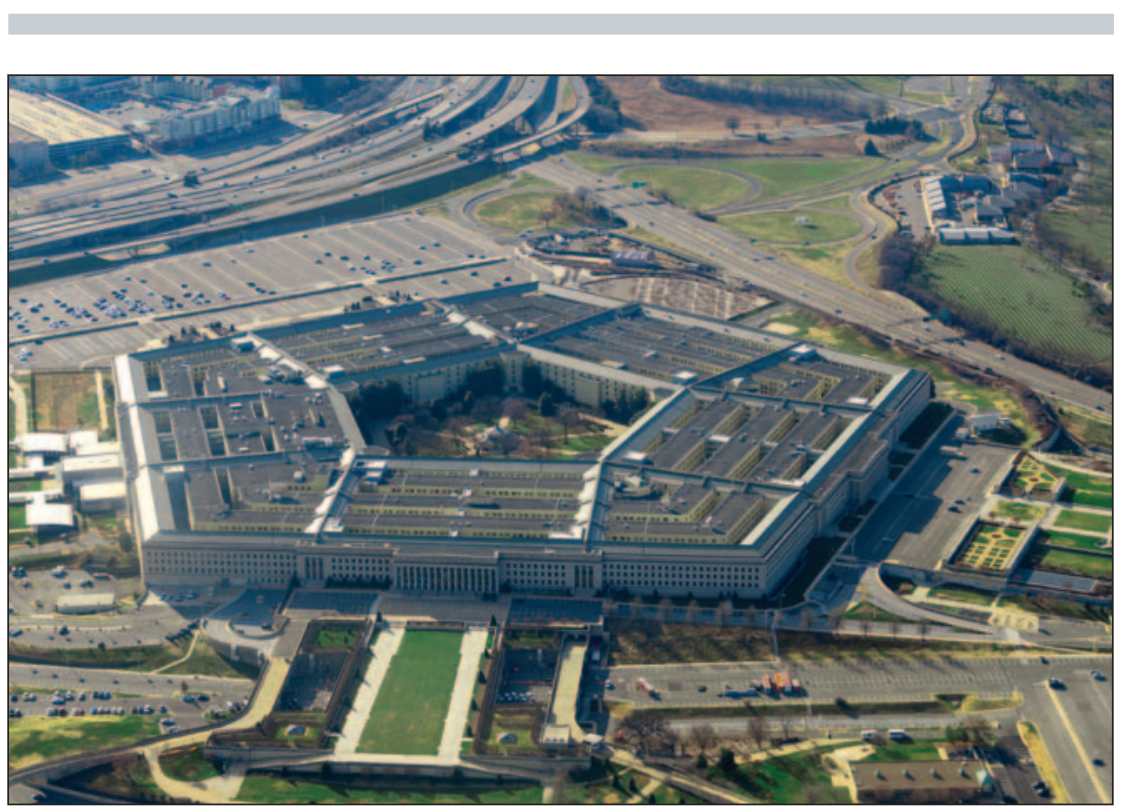

blocks would have other beneficial applications, such as planned journeys to Mars - by both government and the private sector - in which reducing payloads will be critical to success.

Both intramural and extramural research is funded by DoD to the tune of about \$2 billion annually, all of it overseen by BRO. DoD is the third-largest funding source for S\&T research at US universities, after the National Institutes of Health and the National Science Foundation (NSF). Programs include single investigator awards, defense laboratory and university laboratory collaborations, and training for researchers to extend their work beyond the laboratory.

All DoD funding opportunities, including those that BRO oversees, can be found at www.grants.gov. Each opportunity will have the name and contact information for a person associated with that program, says BRO Interim Director Bindu Nair - a materials scientist.

"Awareness of the funding cycles is critically important," Nair says, "because program managers can speak much more freely to an interested PI [principal investigator] before a funding opportunity is posted to grants.gov due to restrictions aimed at ensuring fair competition."

DoD basic research programs are funding efforts at Vanderbilt University to develop and test new composite materials that provide improved blast and projectile impact resistance in protective armor panels; University of Pittsburgh to enable prosthetic limbs to gain the feeling of touch through an array of microelectrodes; and SRI International, where researchers have developed an oral tablet that binds ions of ingested radioactive materials as a possible countermeasure against terror attacks, to name just a very few.

DoD basic research funding is awarded to institutions in all 50 states through such programs as the Vannevar Bush Faculty 
Fellowship support to university faculty and staff scientists to conduct research important to national security; Multidisciplinary University Research initiatives, which support research teams to address S\&T problems that span disciplines; Historically Black Colleges and Universities/Minority Serving Institutions to conduct research and educate scientists and engineers in areas important to national defense; Future Directions Workshops, which connect leading scientists and engineers from academia, national laboratories, and industry to discuss scientific areas of rapid progress and create a roadmap to capitalize on the opportunities created by those advances; and I-Corps@ DoD, a pilot program in partnership with
NSF to provide entrepreneurship training and mentorship.

The January 30 briefing on Capitol Hill was the second of BRO's Science, Technology, and Innovation Exchange (STIx), meant to showcase the impact that DoD S\&T programs have had by providing the primary investigators, program managers, and students a platform to share their research efforts with broad audiences, including the general public. DoD says STIx allows its investments and contributions to S\&T to be illustrated in a way that promotes collaboration and learning.

Nair says early engagement is key for researchers interested in obtaining DoD funding for their research. "Principal investigators should talk to the program officer and engage in technical discussions before a specific opportunity is released," she says. "PIs can use the last broad agency announcement, found on the agency's website, to figure out which program officer they should contact regarding a specific research area."

The goals of BRO, Nair says, include developing and supporting a cadre of science professionals across the United States.

"We want to help researchers figure out how to create fabulous new science," Nair says. "The hope is to continue developing the next generation of researchers for DoD and the country at large."

William G. Schulz

Canada-UK Advanced Manufacturing Partnering Mission identifies collaboration opportunities

Tnternational research and development (R\&D) collaboration is essential for Canadian small- and medium-sized enterprises (SMEs) looking to accelerate their growth and access new markets. As a priority area of focus, the National Research Council of Canada Industrial Research Assistance Program (NRC IRAP) creates opportunities for innovative firms to collaborate and grow beyond Canada's borders.

Recently a delegation of 20 SMEs and industry representatives from the UK traveled to Canada as part of the Canada-UK Advanced Manufacturing Partnering Mission, held in Toronto and Winnipeg from April 16 to 20, 2018. Organized by the NRC in Canada and by Innovate UK and the European Enterprise Network in the UK, the goal of the mission was to create opportunities for the development of joint Canada-UK advanced manufacturing research projects in time to submit project proposals for the September 2018 cutoff for the Eurostars-2 program.
Eurostars-2 provides innovation advantages for Canadian SMEs through access to technology, expertise, and markets in Europe and beyond. The program is aimed at supporting both development and commercialization of new innovative products, services, and processes and helps technology-orientated SMEs to realize the many benefits of working beyond national borders. Canada joined the Eurostars-2 program (2014-2020) as a partner country in March 2016, and has already engaged in a number of projects, including partnership with companies in the UK.

One project, for example, focuses on unconventional or alternative energies serving the market area of oil and gas exploration and production. For this project-started in September 2017the researchers aim to produce coal mine methane to generate energy. The SMEs involved are New Paradigm Engineering Ltd. (Canada), Alkane Energy Plc (UK), and Hofseth BioCare ASA(Norway). This project is called MineGas. Another project, MiDAS, serves the market area of geothermal energy. This partnership between LuminUltra Technologies Ltd. (Canada) and WRc Plc (UK) also began last fall.

In addition to one-on-one meetings and networking sessions in April, the delegation had the opportunity to explore the Canadian advanced manufacturing research landscape through visits to a number of manufacturing and research facilities.

"The Canada-UK mission was a fantastic opportunity to begin forming strong partnerships that can tackle high-value challenges in advanced manufacturing, leading to economic benefits for both our economies," says David Lisk, vice president of NRC IRAP.

Jane Watkins, national programme coordinator for Eurostars with Innovate UK, says, "I'm already getting a feeling that we're going to get submissions in September 2018, which I think will be a good result."

We welcome comments and feedback on these articles via email toBulletin@mrs.org.

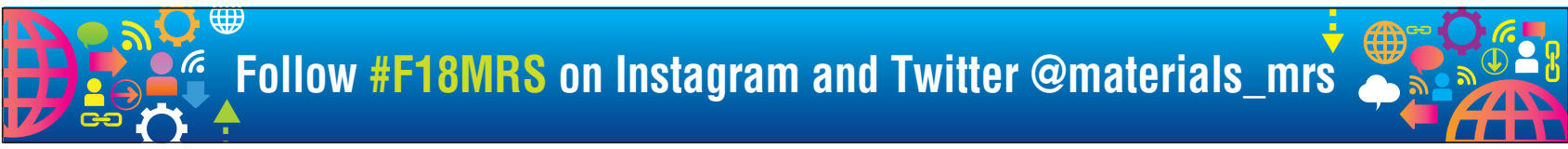

